\title{
Caracterización de situaciones propicias para el engelamiento en aviones
}

https://doi.org/10.31978/639-19-010-0.215

\author{
Alfonso de Miguel Arribas ${ }^{1}$ (alfonso.demiguel.arribas@gmail.com) \\ Juan Pablo Simarro Grande ${ }^{1}$ (jsimarrog@aemet.es)
}

${ }^{1}$ AEMET / Delegación Territorial en la Comunidad Valenciana

\begin{abstract}
RESUMEN
En este trabajo hemos tratado de estudiar el problema de la predicción de engelamiento en aviones. Para ello hemos tenido en cuenta las aeronotificaciones, disponibles desde 2015 aproximadamente. Estas observaciones son escasas y no sistemáticas, ya que son emitidas por los pilotos a ENAIRE por radio, y desde ENAIRE a AEMET a través de correo electrónico. A pesar de la relativa escasez de estas observaciones, son de gran valor, ya que son las únicas observaciones de engelamiento actualmente disponibles en AEMET. El trabajo comprende un exhaustivo estudio de caracterización de las situaciones en las que se produjo engelamiento, desde el punto de vista sinóptico y de situación de frentes. Se han usado también los mapas de baja cota elaborados en AEMET, que incluyen el diagnóstico y predicción de engelamiento. Asimismo se han evaluado campos numéricos procedentes del modelo del ECWMF relacionados con el engelamiento, como la temperatura, la humedad, y distintos hidrometeoros, como el agua subfundida integrada.
\end{abstract}

PALABRAS CLAVE: engelamiento; aeronotificaciones; predicción; modelos numéricos.

\section{INTRODUCCIÓN}

El engelamiento atmosférico ocurre cuando las gotas de agua en la atmósfera se congelan sobre objetos con los que entran en contacto. Este fenómeno es extremadamente peligroso en el contexto de la aviación, ya que la formación de hielo modifica la aerodinámica de las superficies de vuelo, lo cual puede incrementar el riesgo de entrada en pérdida de los perfiles alares de las aeronaves (Schultz y Politovich, 1992).

No todo el agua se congela a $0{ }^{\circ} \mathrm{C}$. El agua líquida por debajo de esta temperatura se denomina agua superenfriada (o subfundida), y es este estado del agua el que causa los problemas de engelamiento en la aeronave. Por debajo de los $-20^{\circ} \mathrm{C}$, el engelamiento es raro ya que a esas temperaturas las nubes consisten usualmente de partículas de hielo en lugar de gotas de agua superenfriada. Por debajo de $\operatorname{los}-48^{\circ} \mathrm{C}$, el agua superenfriada no puede existir, por lo tanto el engelamiento es imposible (MOORE y MOLINERO, 2011).

En aviación, se habla de condiciones de engelamiento a las condiciones atmosféricas que llevan a la formación de hielo en las superficies de la aeronave, o incluso dentro de las turbinas (GóNZALEZ, 2005).

Se identifican varios tipos de engelamiento (BERnsteIN et al., 1997):

- Hielo claro (clear ice). Este es, en efecto, a menudo transparente y liso. Las gotas de agua superenfriada, o lluvia engelante, golpean una superficie pero no se congelan de forma instantánea. A menudo, se forman «cuernos» o protuberancias y se proyectan en el flujo del aire. 
- Hielo blanco o cencellada ( rime ice). Este es áspero y opaco, formado por gotas superenfriadas que rápidamente se congelan al impactar. Se forma mayoritariamente a lo largo del punto de estancamiento de la superficie alar y generalmente se adapta a la forma de tal superficie.

- Hielo mixto. Es una combinación de hielo transparente y de hielo blanco.

- Hielo de helada (frost ice). Este es el resultado de agua que se congela en superficies desprotegidas mientras la aeronave está en reposo. Esto puede ser peligroso porque puede causar una pérdida aerodinámica prematura y, en algunos casos, hacer que el despegue sea peligroso o imposible.

- SLD ice se refiere al hielo formado en condiciones de Supercooled Large Droplet. Es similar al hielo claro, pero como el tamaño de la gota es grande, se extiende a partes desprotegidas de la aeronave y forma estructuras de hielo más grandes, y a un ritmo más rápido que en condiciones de engelamiento normales.

\section{OBSERVACIONES DE ENGELAMIENTO AIREP}

Las observaciones de engelamiento que hemos utilizado corresponden a partes AIREP provenientes de AENA y recogidos de la base de datos de AEMET. Los AIREP dan información sobre observaciones de fenómenos meteorológicos adversos realizadas por los pilotos de las aeronaves y transmitidas a las oficinas de vigilancia meteorológica (OVM). AEMET emite estos mensajes en el formato AIREP(AEMET, 2015). Por ejemplo:

UASP60 LEMM 011629

ARS RYR218 MOD ICE OBS AT 1310Z N3945 W0007 FL150-200,

es un AIREP de engelamiento moderado, observado en la aeronave RYR218 a las 13:10 Z, en las coordenadas geográficas $0^{\circ} 7^{\prime} \mathrm{W}, 39^{\circ} 45^{\prime} \mathrm{N}$, entre los niveles de vuelo 150-200.

La OVM de Valencia emite los AIREP correspondientes a los FIR de Madrid y de Barcelona, y la OVM de Canarias emite los AIREP correspondientes al FIR de Canarias. También se emiten AIREP cuando se observa turbulencia, ondas de montaña, cumulonimbos o actividad volcánica.

En total hemos dispuesto de 124 observaciones AIREP de engelamiento moderado o severo en el FIR de Madrid y de Barcelona. Estos partes abarcan los años 2015, 2016 y 2017, y por tanto, son una fuente de información relativamente reciente.

Los AIREP suponen información muy valiosa tanto para los usuarios de aviación como para AEMET. Desafortunadamente, esta fuente de datos presenta varias limitaciones. La primera es que su número es efectivamente escaso. Otra limitación importante es que, al ser una información generada por el propio piloto, posee más incertidumbre espacial y temporal de lo deseable. Además, no disponemos de observaciones negativas o de ausencia de engelamiento, y por añadidura, la ausencia de observación no implica que no hubiera posibilidad de engelamiento, o incluso que realmente se produjera en alguna aeronave.

En la figura 1 podemos ver la distribución geográfica de todos los partes AIREP. En verde aparecen las notificaciones de engelamiento moderado y en morado las de engelamiento severo. Podemos ver una acumulación en la región de Madrid y en todo el litoral del Mediterráneo y las Baleares.

En la figura 2, a la izquierda, mostramos la distribución de los AIREP por niveles de vuelo. Aquí podemos ver una acumulación de los mismos en niveles medios y bajos. A partir de FL350 la presencia de AIREP es despreciable. En la figura de la derecha tenemos la distribución por estación e intensidad. Para engelamiento moderado, predomina invierno, con una presencia significativa de observaciones durante el resto del año. Para engelamiento severo, la presencia de notificaciones en primavera y verano es notablemente menor. 


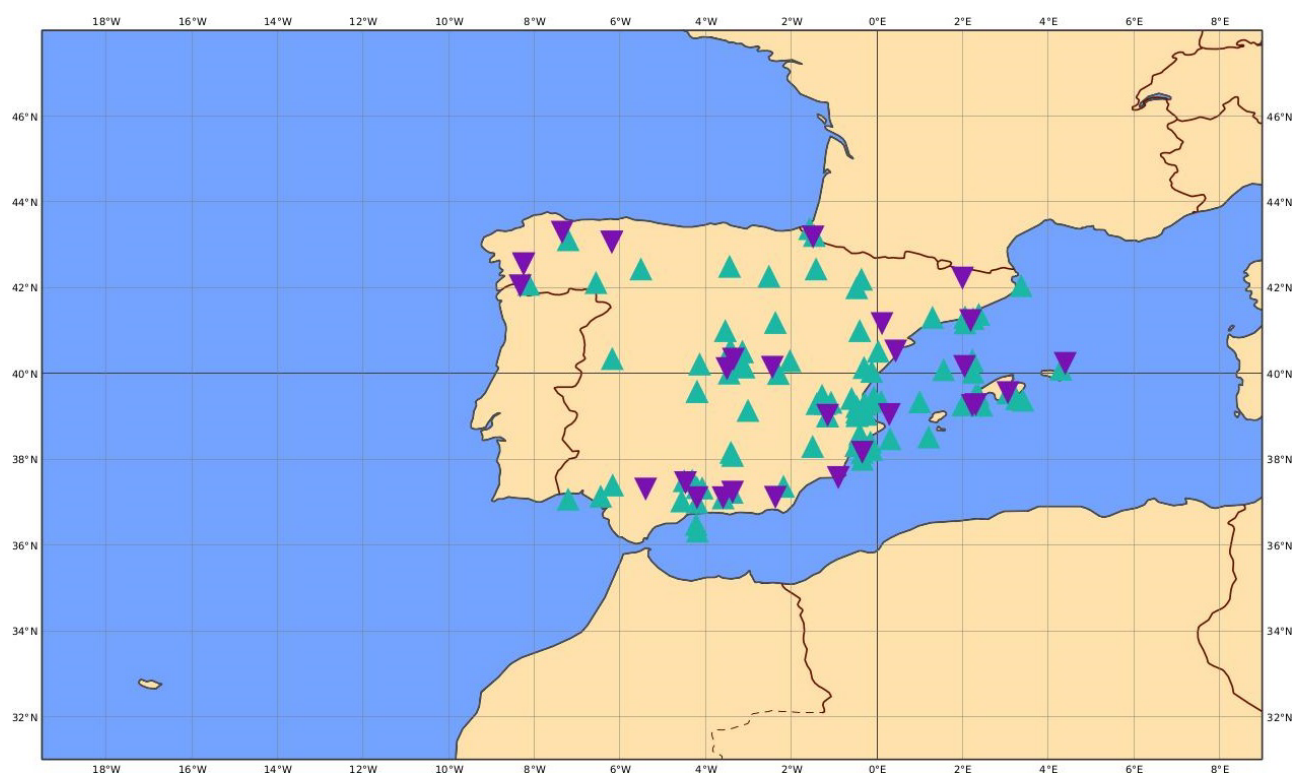

Figura 1. Distribución geográfica de las observaciones de engelamiento moderado (verde) y severo (morado).
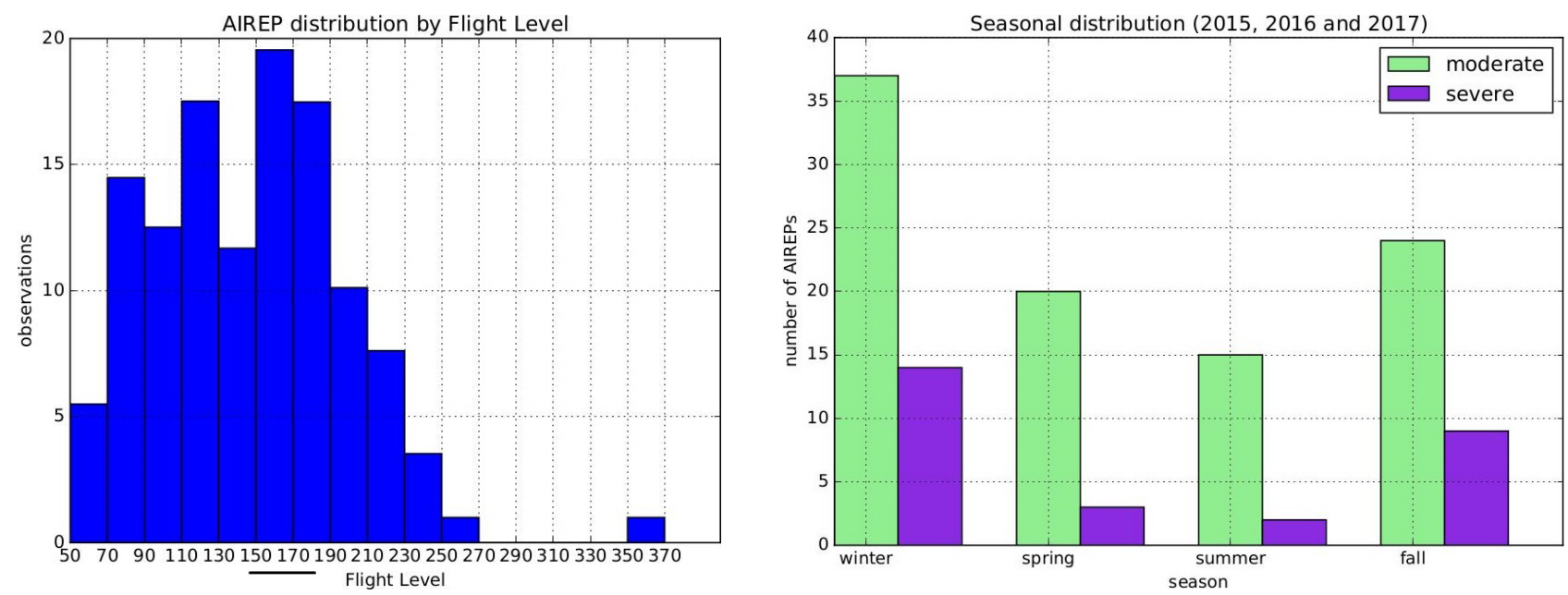

Figura 2. Distribución de observaciones de engelamiento según el nivel de vuelo (izquierda) y las estaciones del año (derecha).

\section{CLASIFICACIÓN POR FRENTES Y SINÓPTICA}

Hemos estudiado, para cada una de las 124 observaciones disponibles, el tipo de frente presente. Para ello hemos revisado los mapas de análisis de superficie correspondientes a cada AIREP. En estos mapas se dibujan los frentes superpuestos a una imagen de satélite en el canal visible, tal como se observa en el ejemplo de la figura 3.

Encontramos una predominancia de paso de frentes fríos (51 casos de 124), seguida de frentes ocluidos (22) y de líneas de inestabilidad (16) (tabla 1). La categoría «otros» comprende situaciones en las que no había señalada la presencia de frente o línea alguna, pero sí había señalización de precipitación o tormentas. La categoría «nada» se asignó a las situaciones en las no figuraba absolutamente nada en el mapa, ni presencia de frentes ni otros fenómenos. Se observó engelamiento en 3 ocasiones en frentes cálidos, y una sola vez en un frente estacionario. En 4 casos no se dispuso de información para hacer esta clasificación. 


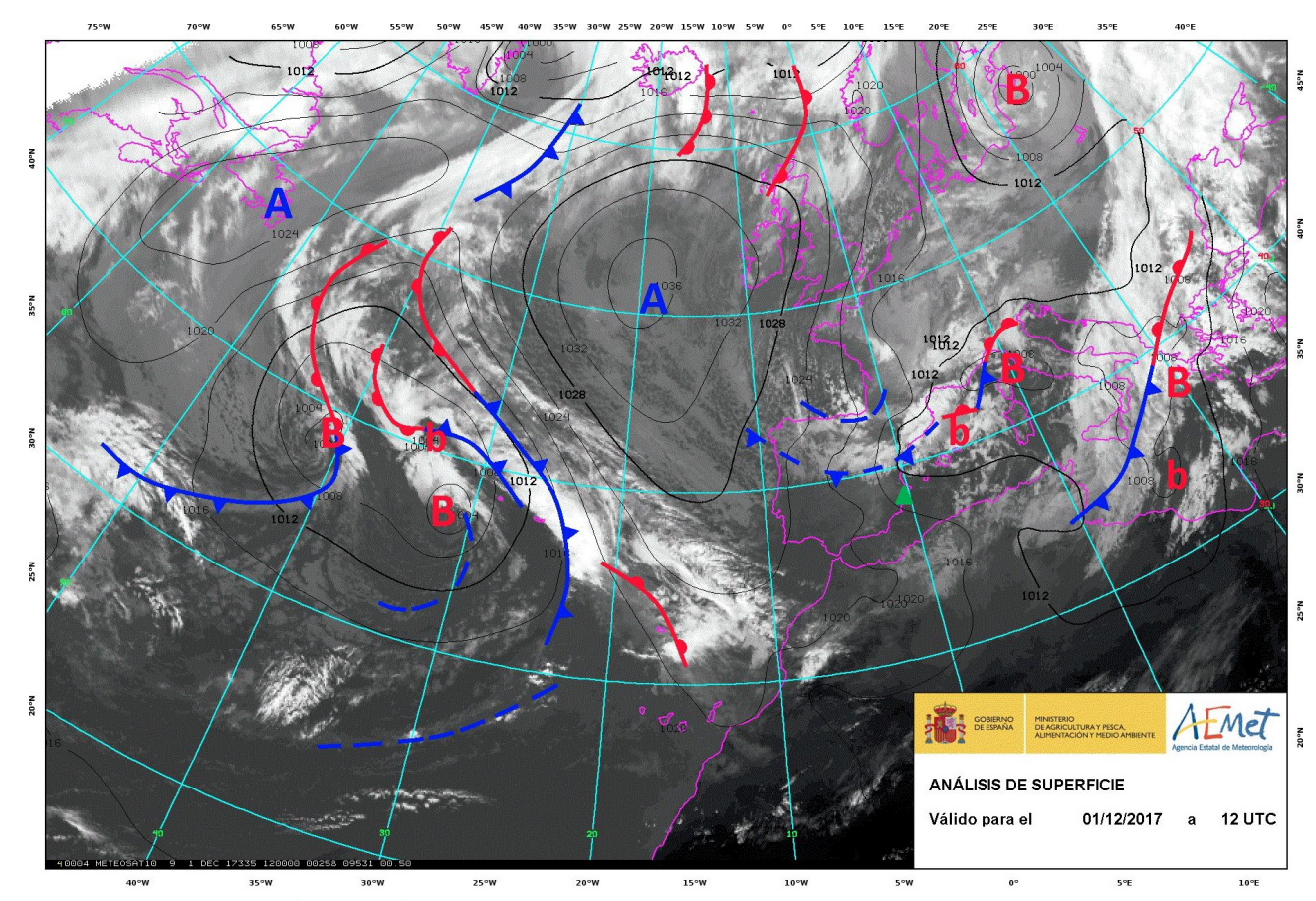

Figura 3. Mapas de análisis de superficie realizados en el Centro Nacional de Predicción, con imagen de satélite visible de fondo. Se ha añadido la observación de engelamiento (triángulo verde).

Para la caracterización sinóptica nos hemos servido de los análisis y predicciones del ECMWF para la altura geopotencial en $500 \mathrm{hPa}$ (tabla 2). Encontramos para la configuración del geopotencial una predominancia de vaguadas (54 casos), seguido en similar importancia de centros de bajas presiones (32) y flujo zonal (30 casos). Son minoritarias, pero no inexistentes, las situaciones con dorsal y otras configuraciones menos definidas.

\begin{tabular}{lc} 
Situación & Frecuencia \\
\hline Frente frío & 51 \\
Frente ocluido & 22 \\
Línea de inestabilidad & 16 \\
Otros & 16 \\
Nada & 11 \\
Frente cálido & 3 \\
Frente estacionario & 1 \\
Sin información & 4 \\
\hline
\end{tabular}

Tabla 1. Clasificación de las observaciones de engelamiento según la situación frontal.

\begin{tabular}{lc} 
Situación & Frecuencia \\
\hline Vaguada & 54 \\
Centros de bajas & 32 \\
Flujo zonal & 30 \\
Dorsal & 4 \\
Otros & 6 \\
Centros de altas & 0 \\
\hline
\end{tabular}

Tabla 2. Clasificación de las observaciones de engelamiento según la situación sinóptica.

\section{COMPARACIÓN CON MAPAS DE BAJA COTA}

Los mapas de baja cota, elaborados en las OVM de Valencia y Canarias para los distintos FIR, contienen predicciones a corto plazo de engelamiento, desde superficie hasta el nivel de vuelo 150 , con validez para una ventana temporal de 6 horas (AEMET, 2015). Vemos un ejemplo en la figura 4. En estos mapas se muestran las predicciones de fenómenos meteorológicos adversos significativos para la aviación. Para nuestro trabajo nos hemos fijado en la presencia o no de los símbolos asociados a engelamiento. También se han tenido en 


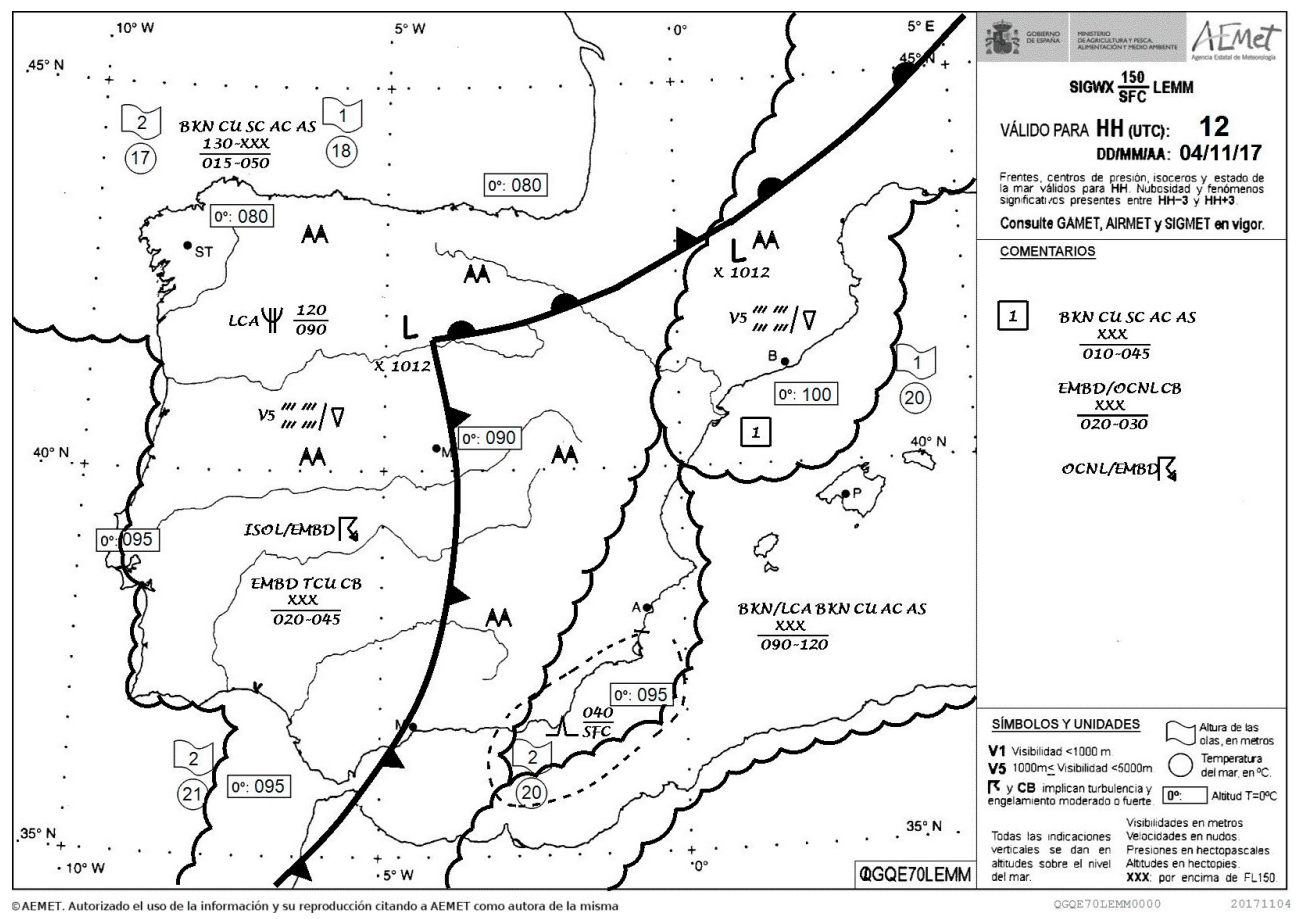

Figura 4. Ejemplo de mapa de baja cota elaborado en la OVM de Valencia.

cuenta los símbolos que indican presencia de tormentas y cumulonimbos, ya que se supone implícitamente que puede producirse engelamiento asociado a convección.

La verificación ha consistido en lo siguiente. Observación por observación, hemos inspeccionado el mapa de baja cota asociado en fecha y hora y hemos buscado la presencia de predicciones de engelamiento, no convectivo o convectivo. Hemos considerado como hallazgo positivo si para una observación dada había al menos una predicción de engelamiento en algún punto del mapa. Además hemos valorado de manera cualitativa la proximidad de la observación a la región de la predicción, clasificándola en tres categorías: dentro, cerca y lejos, según la observación de engelamiento se hallara dentro, cerca o lejos de una zona con predicción de engelamiento convectivo o no convectivo.

Hemos encontrado los siguientes resultados, resumidos en la tabla 3. En el $94 \%$ de AIREP con FL por debajo de 150, hay predicción de engelamiento en el mapa de baja cota pertinente. En cuanto a la proximidad observación-predicción, estos son los resultados. Cuando en el mapa había únicamente predicción de engelamiento no convectivo, un $30 \%$ de los casos se hallaban dentro del área festoneada, y un $50 \%$ se hallaban razonablemente próximos. Para engelamiento convectivo, el número de casos dentro del área con predicción de tormentas o cumulonimbos asciende al $37 \%$, aunque la suma de las categorías dentro y cerca es prácticamente igual que en el caso anterior, $80 \%$. Cuando aparecen ambos tipos de predicción en el mapa, el número de casos dentro de zonas de predicción asciende hasta el $71 \%$, y la suma dentro y cerca supone ahora un $93 \%$.

\begin{tabular}{lccc} 
Observación & Dentro (\%) & Cerca (\%) & Fuera (\%) \\
\hline Engelamiento & 30 & 50 & 20 \\
Cumulonimbus / tormentas & 37 & 44 & 19 \\
Engelamiento / Cumulonimbos / tormentas & 72 & 21 & 7 \\
\hline
\end{tabular}

Tabla 3. Clasificación de las observaciones de engelamiento por debajo del nivel de vuelo 150 en función de la predicción de engelamiento de los mapas de baja cota. 


\section{CAMPOS NUMÉRICOS DEL MODELO HRES-IFS DEL ECMWF}

Hemos obtenido de la base de datos MARS del ECMWF diversos campos relacionados con el engelamiento (IsAAC et al., 2001): temperatura y humedad relativa en diversos niveles isobáricos, nubosidad en distintos niveles, índices de inestabilidad como CAPE o K, hielo integrado en la vertical, agua líquida y agua líquida subfundida integrada en la vertical, entre otros campos.

Se ha realizado una revisión cualitativa y pormenorizada de la respuesta de estos campos en el entorno espaciotemporal de cada una de las observaciones. Encontramos, en la gran mayoría de situaciones, una situación física y teóricamente razonable para la formación de engelamiento. Sin embargo, en unos pocos casos, no se ha podido encontrar una justificación mínimamente favorable al engelamiento.
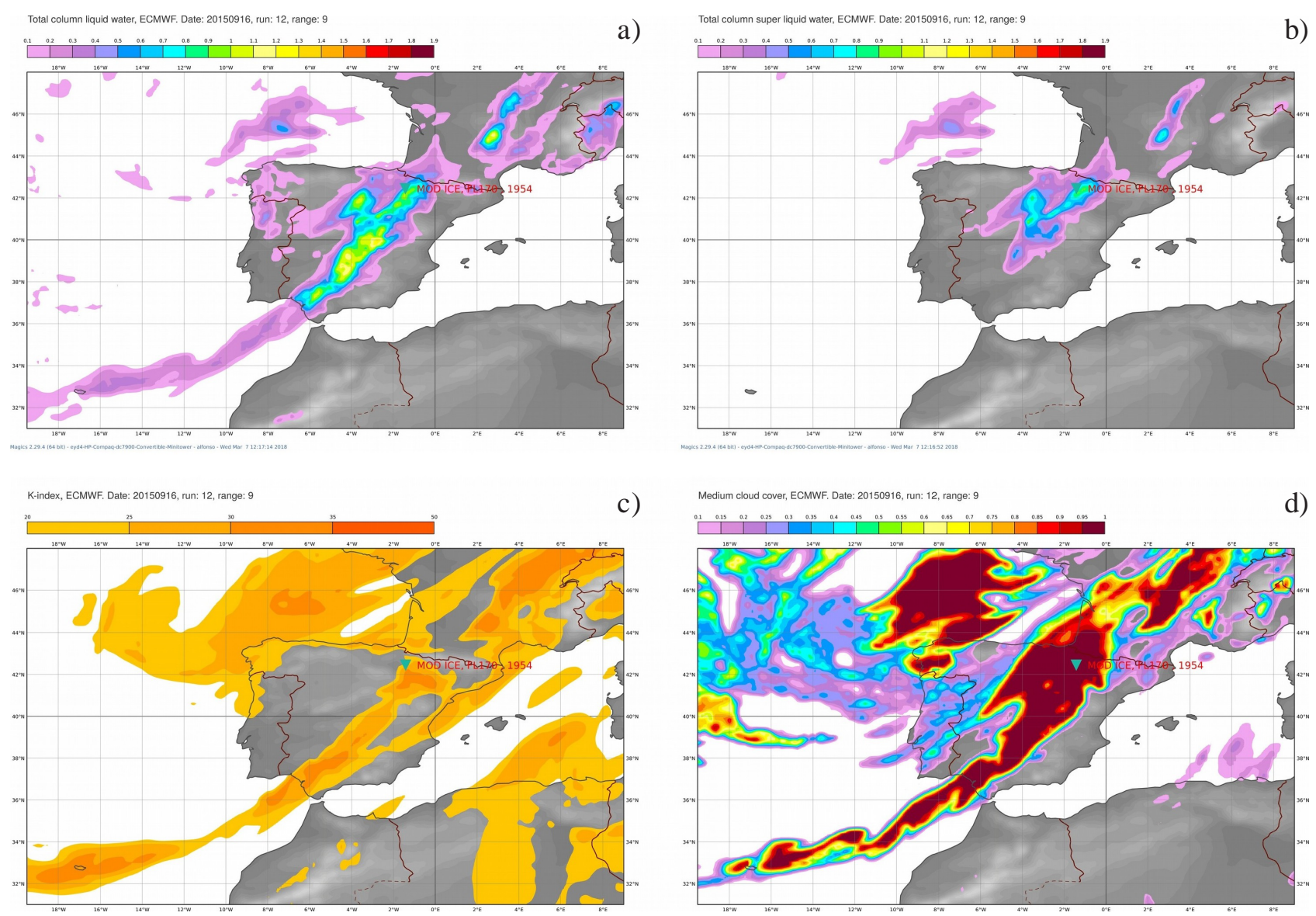

Figura 5. Ejemplo con algunos de los campos numéricos del ECMWF utilizados, con la observación de engelamiento superpuesta. a) Total de agua líquida integrada en la columna, b) total de agua subfundida integrada en la columna, c) índice $\mathrm{K}$ y d) cobertura de nubosidad media.

Para complementar este análisis, se procedió a un estudio de tipo cuantitativo. Dado un campo de interés en la predicción de engelamiento, por ejemplo, el contenido de agua subfundida integrado en la vertical, tomamos los alcances en torno a cada observación en un área centrada en la península ibérica (el área que aparece en la figura 5). Con los valores que toma ese campo en los puntos de rejilla de esa amplia área se construye un histograma, es decir, la distribución que tiene dicho campo en un entorno de la península ibérica en las horas en las que se observó engelamiento. A este histograma lo denominamos histograma de fondo. Es importante resaltar que esta distribución obedece a valores siempre dentro de una situación de observación de engelamiento. Para el mismo campo y mismos alcances, se seleccionan los valores de la rejilla limitándonos a un radio espacial de $150 \mathrm{~km}$ en el entorno de la misma. De esta forma se construye lo que denominamos 
el histograma total in situ. Este histograma describe los valores que toma el campo en estudio en las cercanías de los lugares en los que se observó engelamiento, frente al histograma de fondo. Para que ambos histogramas (el de fondo y el in situ) puedan compararse, se normalizan.

a)

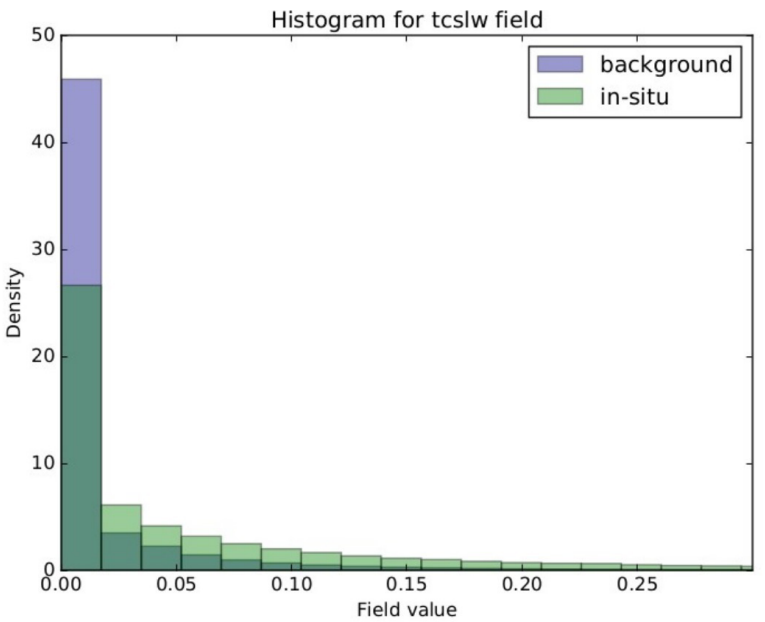

c)

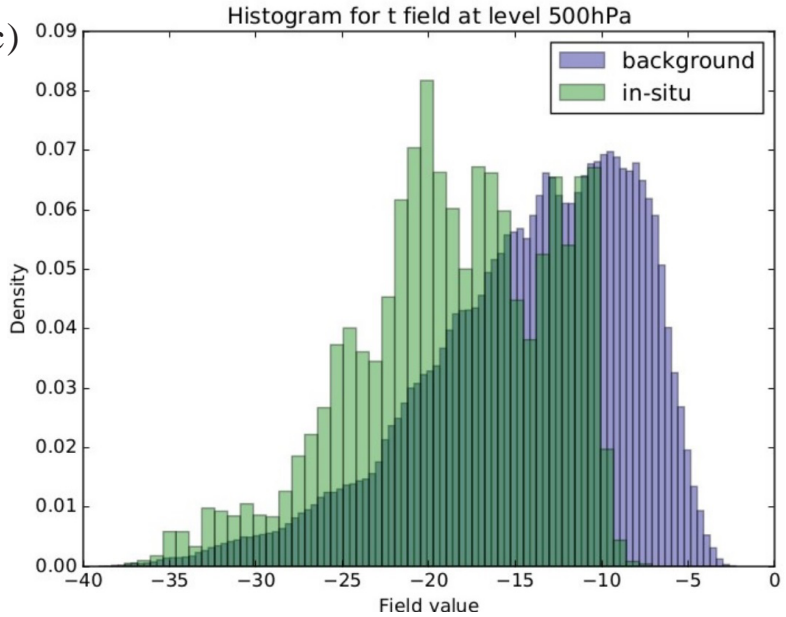

b)

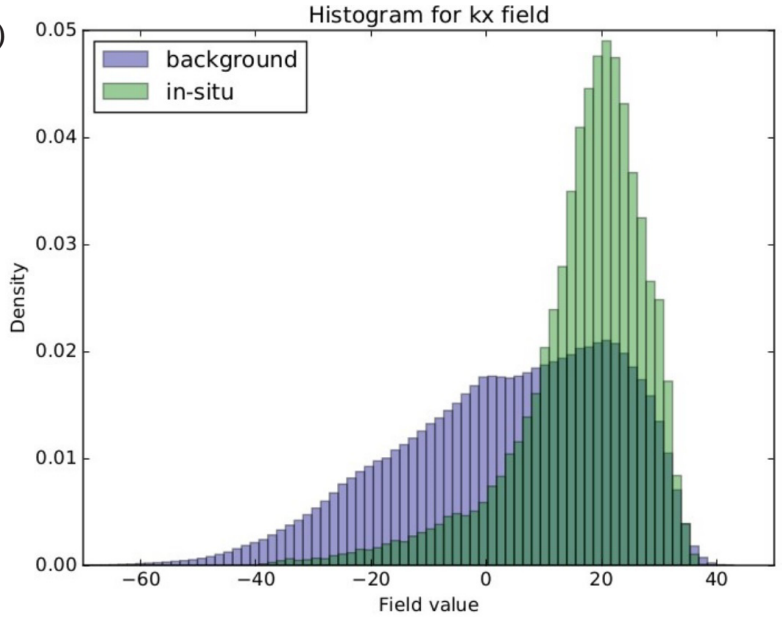

d)

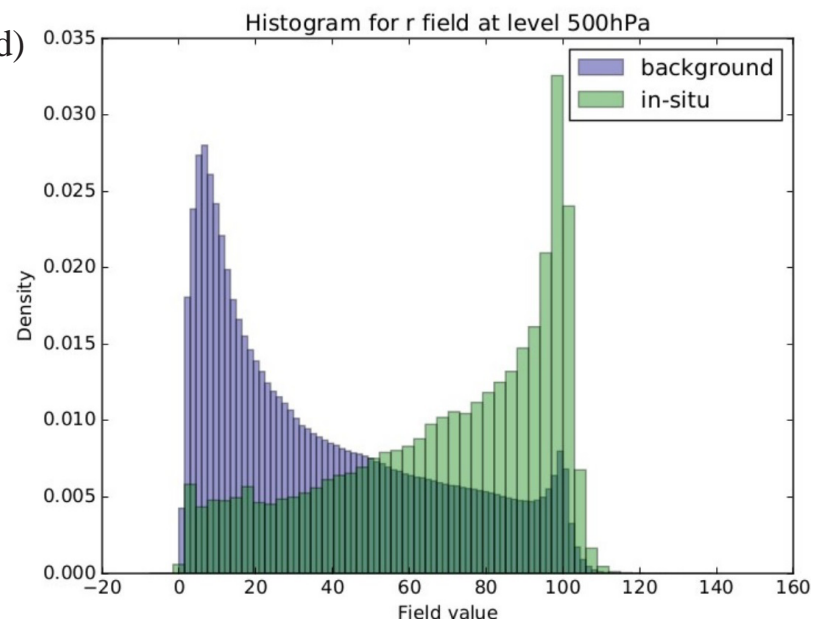

Figura 6. Comparación entre los histogramas in situ y los histogramas de fondo para algunos campos meteorológicos del ECMWF utilizados. a) Agua subfundida integrada en la vertical,

b) índice de inestabilidad $\mathrm{K}, \mathrm{c}$ ) temperatura en $500 \mathrm{hPa}$ y d) humedad relativa en $500 \mathrm{hPa}$.

Seleccionamos algunos de los resultados obtenidos, y los mostramos en la figura 6. En esta figura podemos ver los histogramas de fondo e in situ para el agua subfundida integrada en la vertical, el índice de inestabilidad K, la temperatura en $500 \mathrm{hPa}$ y la humedad relativa en $500 \mathrm{hPa}$. Se observa claramente la diferencia entre las distribuciones para todos los casos, algunos más destacados que otros. Así por ejemplo, la humedad relativa en $500 \mathrm{hPa}$ tiene dos histogramas muy diferenciados, estando el máximo de la distribución in situ en valores en torno al $100 \%$ de humedad relativa, mientras que la distribución de fondo tiene el máximo en valores muy bajos, cercanos al $10 \%$. Para la temperatura en $500 \mathrm{hPa}$ la discriminación entre distribuciones sigue siendo evidente, aunque no tan marcada como en el caso de la humedad relativa. Aquí vemos cómo la distribución in situ está desplazada hacia la izquierda, encontrando valores muy altos desde $-10^{\circ} \mathrm{C}$ hasta $-20^{\circ} \mathrm{C}$. Los resultados para el valor integrado del agua subfundida, que sería una medida directa de engelamiento, también muestra una clara diferencia entre las distribuciones. Los valores altos de esta variable son más frecuentes en las cercanías de la observación (histograma in situ), mientras que los valores bajos son más frecuentes en el histograma de fondo. Para el índice K, asociado a inestabilidad, vemos de nuevo la diferencia de distribuciones de fondo e in situ. Esta última tiene un pico de gran peso en torno a valores que indican probabilidad de inestabilidad. Por tanto, en todos los casos mostrados en la figura 6 se obtiene lo esperado: en las cercanías de las observaciones de engelamiento hay más humedad relativa, las temperaturas son más bajas, hay más inestabilidad y mayor cantidad de agua subfundida. 


\section{CONCLUSIONES}

A partir de la interesante información aportada por los partes AIREP, y con el fin de lograr avances en el problema de la predicción del engelamiento, decidimos realizar un estudio sistemático sobre este fenómeno en aviones. Nos servimos de las 124 observaciones de engelamiento disponibles de los periodos 2015, 2016 y 2017 en la península ibérica.

El resumen de resultados del análisis es el siguiente. Se han tratado de caracterizar las situaciones según la existencia o no de frentes (fríos, cálidos, ocluidos, líneas de inestabilidad y otros). Las situaciones más frecuentes son las de frentes fríos, ocluidos y líneas de inestabilidad. De forma similar se han caracterizado las observaciones según las situaciones sinópticas (bajas, altas, vaguadas, dorsales, flujo zonal y otras). Las situaciones más frecuentes son las de vaguadas, centros de bajas y flujo zonal.

A continuación se ha realizado una comparación entre las observaciones y las predicciones de engelamiento que aparecen en los mapas de baja cota elaborados en las OVM. Se observa en general una buena correlación entre las predicciones y las observaciones.

Finalmente se han usado campos meteorológicos del ECMWF relacionados con el engelamiento, como pueden ser nubosidad, temperatura y humedad en distintos niveles, índices de inestabilidad, o hidrometeoros integrados en la vertical (en particular el agua subfundida). Este estudio se ha hecho de forma cualitativa y cuantitativa. En el estudio cuantitativo se han comparado los histogramas de fondo con los histogramas in situ, y para algunas variables se observa que estos son claramente distintos, indicando el valor predictivo de estas variables.

Los resultados expuestos en este texto son un resumen del trabajo realizado, que por cuestión de espacio no se ha podido exponer en su totalidad.

\section{REFERENCIAS}

Agencia Estatal de Meteorología (AEMET), 2015. Guía Met: Información Meteorológica Aeronaútica. AEMET, 12. ${ }^{\text {e edición. }}$

GoNZÁLEZ LóPEZ, B., 2005. Meteorología aeronáutica. AVA. ISBN 849337203X, 9788493372033.

Schultz, P. y Politovich, M. K., 1992. Toward the improvement of aircraft-icing forecasts for the continental United States. Weather and Forecasting, 7, 491-500.

IsAAC, G. A. et al., 2001. Recent Canadian research on aircraft in-flight icing. Canadian Aeronautics and Space Journal, 47 (3), 213-221.

BERnSTEIN, B. C. et al., 1997. The relationship between aircraft icing and synoptic-scale weather conditions. Weather and Forecasting, 12, 742-762.

Moore, E. B. y Molinero, V., 2011. Structural transformation in supercooled water controls the crystallization rate of ice. Nature, 479 (7374), 506-508. 